\title{
Crosscultural narratives on death and bereavement among medical students: implications for undergraduate curricula
}

\author{
Arokiamary Bharathy ${ }^{1}$, Margarita Malayapillay ${ }^{2}$, Vincent Russell ${ }^{1}$ \\ ${ }^{1}$ Department of Psychiatry, Penang Medical College, Malaysia \\ ${ }^{2}$ Penang Association of Counselling and Psychology, Malaysia
}

Correspondence: Arokiamary Bharathy, Department of Psychiatry, Penang Medical College, Malaysia. Email: bharathy@pmc.edu.my

\begin{abstract}
Objectives: The aims of this study were to explore how Asian medical students from different cultural backgrounds engaged with issues of death and bereavement in an interactive group setting and to report their subjective experience of participation in the group.

Methods: A convenience sample of thirteen, fourth year students at Penang Medical College participated in an audio-recorded focus group. Thematic analysis was performed on their personal narratives on death and bereavement.

Results: The dominant themes that emerged were openness with regard to students' narratives, strong identification with traditional beliefs and rituals, the influence of profes
\end{abstract}

sional identity and recognition of commonality of bereavement experience. There was some discomfort arising from the group process but this was mitigated by the level of support within the group.

Conclusions: The implications of our findings for undergraduate medical curricula are that interactive, small group teaching of this important but culturally sensitive topic would be well tolerated and acceptable in an Asian medical school setting. However, the group process must be carefully structured and facilitated.

Keywords: Bereavement, crosscultural, medical students, undergraduate curricula

\section{Introduction}

Cultural competence is recognised as necessary quality among health professionals and an essential component of medical education. ${ }^{1}$ Over the past decade there has been significant progress, particularly in the United States, resulting from collaboration between medical schools to support the implementation and standardisation of cultural competency training. ${ }^{2}$ Nonetheless, from a broader international perspective, the goal of providing widespread cultural competency curricula, linked with improved patient outcomes remains challenging. ${ }^{3,4}$

A doctor's cultural competence is particularly tested in interactions with patients and relatives around the time of death. Heightened emotional sensitivities at this time are such that the doctor's communication and behaviour are likely to have a more significant impact. Effective communication will be determined in part by the doctor's personal experiences of loss and bereavement and also by his or her understanding of the patient's and family's position on death in terms of their ethnic and religious background. ${ }^{5}$ Doctors need to respond in a consistent and sensitive manner irrespective of their own ethnic and religious background or that of the patient. There is also a risk of cultural stereotyping and ethnocentric bias, so that knowledge of cultural practices is not sufficient unless accompanied by attitudes of flexibility and openness. ${ }^{6}$

A distinction has been drawn between cultural competence and cultural humility in that, the application of cultural competence in itself may contain hidden assumptions that define culture by the extent to which the "other" is different from "us" ${ }^{7,8}$ Particular differences have also been described between Western and non-Western conceptualisations of grief and bereavement. In the former there is an emphasis on the finality of death and on grief as a shortlived, individual and private experience. In contrast, traditional cultures tend to view death as a transition between the material and spiritual worlds. Here, grief is conceptual- 
ised as socially negotiated, openly expressed, protracted and facilitated by communal participation in rituals. ${ }^{9}$

End of life training in medical schools has been criticised as too often supplementary rather than embedded in the curriculum and as continuing to rely excessively on lectures and seminars. However, when it is integrated in the curriculum and particularly when it incorporates students' personal experiences of loss, the learning benefits appear to be maximised. ${ }^{10,11}$

Small, interactive groups, incorporating elements of role-play, group discussion and reflective narrative writing have been successfully incorporated into undergraduate curricula. ${ }^{12}$ Culturally informed guidance has also emerged on the teaching of end of life issues. ${ }^{13}$

Such experiential approaches inevitably involve, open discussion of students' cultural beliefs and contentious issues may emerge as a result. These tensions may be further magnified in educational settings within mixed ethnic communities, where rituals and religious practices surrounding death and bereavement strongly reflect core identities and therefore have greater salience and wider observation. $^{14}$

No previous research, to our knowledge, has specifically explored the viability and acceptability to students of interactive, small group educational approaches addressing personal experiences of death and bereavement in an Asian medical school setting. Against this background, we carried out a qualitative study in a Malaysian medical school with a multi-ethnic and multi-religious student representation. The study's aims were twofold: first, to explore how students from different cultural backgrounds engaged with issues of death and bereavement in an interactive group setting and secondly to report their subjective experience of participation in the group itself. We present our findings and discuss the implications for curriculum development in similar settings.

\section{Method}

\section{Setting}

The study was undertaken by the Department of Psychiatry, Penang Medical College (PMC). The college was established as a partnership with two Irish universities, the Royal College of Surgeons in Ireland (RCSI) and University College Dublin (UCD). PMC students undertake their preclinical studies at either RCSI or UCD in Dublin, after which they return to pursue their clinical training in Penang. Each year an average of 120 students rotate in classes of 30 through four eight-week clinical postings in psychiatry.

\section{Sampling procedure}

The study involved a convenience sample from a class of thirty, fourth year students during their posting in psychiatry. The whole class was initially briefed jointly by the first author, $A B$, a Malaysian psychiatrist and full time senior lecturer in the Department of Psychiatry, PMC and the second author, MP, a Malaysian registered licensed counsellor in independent practice. The study was timed to begin at the end of the eight week placement in psychiatry, to minimise disruptions to students' schedules. The class was informed that the study would involve a two hour long focus group exploration of shared narratives in order to understand how participants dealt with death and bereavement issues in a group setting. It was made clear that students' agreement to participate or not, would have no impact on their evaluations and that no incentives or rewards for participation were being offered.

The class was informed that the focus group would be made up of the first 10-15 students who agree to participate. It was felt that given the demographic make-up of the class, this number of participants would be likely to achieve a multi-ethnic, multi-religious representation while yet being small enough for a focus group.

All students were provided with a printed information sheet that addressed the study's aims, confidentiality, the structure and process to be employed, how information would be recorded and processed and the findings disseminated. The information sheet included a questionnaire comprising 10 questions regarding students' previous experiences of bereavement. The questions were drawn up by the first two authors and guided by their professional experience. Ethical approval for the study was obtained from the Joint Penang Independent Ethics Committee.

\section{Participants}

The authors communicated with the class representative one week after the initial briefing and received confirmation that 13 students, 3 male and 10 female had agreed to participate. The participants' ethnic backgrounds and religious affiliations were as follows: six Malay students (all Muslim), six Chinese students (3 Buddhist, 2 Christian and 1 Taoist) and one of Indian origin (Sikh). All participants were single and aged between 24-25 years.

This reflected the demographics of the class as a whole in terms of ethnicity and religion but not gender, as relatively fewer males volunteered. Informed consent was obtained from the thirteen participants. In order to address issues of safety and well-being of the study participants, the focus group facilitators provided their contact details and advised that participants could contact either $\mathrm{AB}$ or MP directly if they experienced distress or felt they needed emotional support as a consequence of the group experience.

\section{Focus group}

The focus group was carried out in a room at the college and facilitated by the first and second authors (AB and MP). Participants were seated at a conference table with fixed microphones so that the session could be audio-recorded. The primary role in leading the group discussion was taken 
by $\mathrm{MP}$ with $\mathrm{AB}$ acting as observer of the group process. The participants were reminded that they would be invited to share their narratives based on the questions provided and that they were free to decide what and how much they wanted to share. They were also informed that they could withdraw from the group at any time during the session without the necessity to give reasons. Participants were requested to respect confidentiality by confining discussion of their personal accounts to the group.

Table 1. Profile of 13 medical students of Penang Medical College in 2011

\begin{tabular}{lc}
\hline Variable & $\mathrm{N}$ \\
\hline Age in years & 6 \\
24 & 7 \\
25 & 3 \\
Gender & 10 \\
Male & \\
Female & 6 \\
Race & 6 \\
Malay & 1 \\
Chinese & \\
Indian & 6 \\
Religion & 3 \\
Muslim & 2 \\
Buddhist & 1 \\
Christian & 1 \\
Taoist & \\
Sikh & \\
\hline
\end{tabular}

They were also advised of other ground rules that sought to provide a respectful, non-threatening atmosphere conducive to sharing what might be personal and painful. Participants were invited to seek clarification from one another where necessary but not to challenge other participants' beliefs. They were reminded that it was desirable to share the available time so that all participants could contribute.

The focus group was conducted in two parts. In the first, participants were invited to share their personal narratives guided by questions 1-9 of the questionnaire, progressing from one participant to the next in turn until all had finished.

During the second part, participants were asked to respond to question 10, which explored how the experience of participating in the group might influence the manner in which they dealt with death and bereavement in the future.

\section{Data analysis}

Because of the exploratory nature of the study the authors agreed to approach the qualitative data from an inductive position. A thematic analysis was undertaken of the focus group transcripts defined as "a coherent way of organizing or reading interview material in relation to a specific research question". ${ }^{15,16}$ In addition, hand-written notes and observations were recorded by the group facilitators.

The transcripts were initially read and re-read by $A B$ and MP to obtain an overall sense of the meaning of the participants' narratives in light of the study's aims. Following this they met to discuss their initial interpretations and agreed on emerging dominant themes. They then re-read the transcripts line by line, marking statements and phrases from the students' narratives, isolating emergent patterns, commonalities and differences, attending both to the manifest and latent content and categorising units of text using the cut and paste word-processing function. ${ }^{16}$

The two authors $\mathrm{AB}$ and MP met again to compare their analysis, addressing points of agreement and divergence while also considering the extent to which relevant data might have been excluded or irrelevant material included. The third author, VR, head of the Department of Psychiatry, PMC, who had no involvement in the focus group itself reviewed the transcripts separately and met with $A B$ and MP to discuss their analysis seeking clarification of their interpretation of the data before all three authors reached consensus.

\section{Results}

\section{Narratives of bereavement experiences}

Openness

Of the thirteen participants, nine recounted experiences of being bereaved of a grandparent, three of another seconddegree relative and one described the loss of a close family friend. None had lost parent or sibling. Most participants provided rich accounts based on personal experiences of bereavement within their family or close friends. There was an obvious willingness on the part of the students to share their stories of loss although frequently painful. A sense of openness and an apparent absence of inhibition or selfcensoring on the part of the students was evident. Participants conveyed honesty of expression and spontaneity, as conveyed in the following quote.

\footnotetext{
"I was supposed to take care of my grandmother because I had school holidays at that time, but then there were some circumstances that I cannot do it and then finding that she was $d y$ ing.... Actually I didn't..., I never cried about this because I never really thought about this but that's it. Thanks." (Student No.3, Female, Malay)
}

For several students it was evident that the group involvement facilitated expressions of intense and sometimes unresolved issues in relation to the loss of loved ones. One student poignantly described how her aunt, to whom she was very close, died at a relatively young age while the student was sitting for college exams in Dublin.

\footnotetext{
"After I received news I was numb about it. I didn't tell anyone about it. Quite a surprise to me that I didn't shed a single tear. I had sleepless nights for one to two months. Only after couple of months I only shared with my friend, first person I told about it. Even after two years it's still affecting me so much...., it changed my view of life. It wouldn't last long, she was not married, no boyfriend, she didn't get to experience a lot of stuff she could have." (Student No.11, Female, Malay)
} 
However, expressions of grief and positive feelings towards the deceased were not entirely uniform. Some participants described the absence of a subjective sense of loss and departed from normative expectations as in the following contribution.

"People might think I'm being quite cold for saying this because he was after all my grandfather, but how can you be sad losing somebody whom you are not close to at all and who ended up teasing you as a child, so I didn't really care about him at all. If I did lose my mum or my siblings, I would be really upset, but it was quite insignificant for me." (Student No.10, Female, Malay)

\section{Identification with traditional beliefs and rituals}

Several participants outlined the religious rituals and cultural practices surrounding death and bereavement associated with their own religious affiliation, often in great detail. In doing so there was an obvious tone of respect and reverence. This pattern was evident in contributions from participants of different religion.

\begin{abstract}
"Actually the ceremony begins with the showering of deceased usually conducted by the family members of same gender or any religious authority of the same gender. It is to preserve the dignity of the deceased. And then after that the body will be covered by white clothes. For women it is three for layers used since the shape of body might be very different from the men." (Student No.2, Female, Malay)
\end{abstract}

"The memorial service was held in the church mortuary and my church pastor came and gave bible reading every night for a period of four days, and my oldest uncle gave a eulogy just one day before he (student's grandfather) was due to be cremated." (Student No.7, Female, Chinese)

"The person will be told to follow the brightest light, or the religious figure like Buddha or Bodhivista, he or she can see and the person needs to let go of earthly issues and beloved family. After eight hours of death, the deceased's body will be bathed and put in clean clothing to look respectable. We wait for eight hours because we believe the soul would take up to eight hours to leave the body, and the soul would be greatly sensitised to all senses like touch and sound and so might perceive touch to body as pain so family members shouldn't cry aloud or scream at bedside so that deceased will not be influenced emotionally and stay on refusing to move on through the journey to afterlife." (Student No.8, Female, Chinese)

There were frequent statements suggesting that students experienced the religious rituals as personally helpful in facilitating the grieving process. Participants valued these rituals because of how they facilitate coping with the loss and moving on with life. Several participants described how burial and commemoration ceremonies had helped family members, bringing them closer together and fostering a sense of mutual support.

"Every year we will visit his tablet in the crematorium during the first day of Chinese New Year and his anniversary which is in May, and even though it has been 5 years since his death. I can still sense the loss in my family and going to see my grandfather... Actually I look forward very much going to see him. Because I believe that my grandfather is in heaven and he is watching over all my family, as all the cousins grow up and go to university and watching over all my aunties and uncles." (Student No.7, Female, Chinese)

Individual group members also expressed a minority view in relation to the significance of traditional customs and rituals surrounding death.

"I think that if we don't really understand about the religious ceremonies or the cultures of the funeral, it is more important to be able to say goodbye properly, emotionally and make peace with death in our own way. It does not have to be a religious or cultural way." (Student No.8, Female, Chinese)

\section{Death as a transition}

An overarching and transcendent theme emerged in many of the students' narratives that the soul of the deceased person goes to a better place and that death is not an end but the beginning of new life. Several Muslim participants highlighted this aspect in their narratives describing death as simply a transition to another life which helped to mitigate the emotional distress and suffering among those bereaved.

\footnotetext{
"The thing I would like to stress is that for Malays especially, religion is actually quite helpful in helping you deal with bereavement and death especially if we remember it's natural that happens to everyone. We are moving on from transient to something that is everlasting. That will probably help to console us." (Student No.5, Male, Malay)
}

"Life on earth for us Muslims is transient, no one will live forever, it will happen to everyone. I think that is the way it will help to console you, to recover from the loss." (Student No.5, Male, Malay)

Buddhist participants echoed this theme, describing how the recitation of prayers brought peace to the deceased as well as calmness and strength while facilitating the transition of the deceased into the afterlife.

\footnotetext{
"I believe quite strongly that if I chant the mantra hard, it can actually send the soul to Heaven and this helped in coping with the loss." (Student No.9, Female, Chinese)
}

\section{Bereavement and professional identity}

There was evidence from a number of students' narratives that the manner in which participants processed the experience of bereavement at both an intellectual and emotional level was affected by their professional identities as medical students and future doctors. In some situations the demands of their studies seemed to impact on the time they felt they had available, to grieve or reflect on the loss. 
"How long did I feel the loss? I coped quite fast. After the funeral I had to come back and resume with my studies so there wasn't really time to think about it, so I guess that helped me to cope with it." (Student No.1, Female, Chinese)

"I only met him during major holidays, soon after two days after that I had to go back to Ireland, to continue my studies so that helped to take my mind off it." (Student No.5, Male, Malay)

In other instances, the medical identity and knowledge seemed to have created an added burden of responsibility for the students, complicating the experience of emotional loss with questions about the medical care afforded to the deceased and opportunities for medical interventions that might have been missed.

"They brought him to GP but the GP actually told them it was nothing serious, so my grandfather went back home. He gradually developed shortness of breath within a few hours they rushed him to Accident and Emergency in a private hospital and then they found out he actually had a heart attack. After that too late... He was scheduled to do a bypass surgery two days later, but he actually died just six hours before his scheduled surgery which came very suddenly to my family... and I remember during that period, that one week of grieving I actually was quite numb. I felt myself thinking about many scientific facts, relying on science to explain my grandfathers' death, rather than relying on religion". (Student No.7, Female, Chinese)

"Suddenly he was gone. I could see it was hard for his direct family and wife because they did not expect it. I was a medical student, when I heard the circumstances of his death I suspected he had a heart attack, chest pain, nausea, felt like vomiting but the symptoms were not recognized by family. I felt a bit frustrated." (Student No.5, Male, Malay)

\section{Impact of the group experience}

\section{Commonality of experience}

In the second phase of the interview, students were asked to describe how they felt about participating in the group. They identified similarities and parallels between various religious rituals and cultural practices.

\begin{abstract}
"Just after hearing everybody's account, I actually do realise that the religious practices were really important. Because somehow after having done all those it makes people accept death more readily, at least they know they have done something for the dead." (Student No.1, Female, Chinese)
\end{abstract}

This sentiment was echoed by several other participants and some went beyond this to articulate the potential benefits of more awareness of other religious and cultural practices.

"As I heard the stories, most religions you know have their similarities on how they handle the deceased, how they handle the body and I personally would like to know more about other religions, so that in case if I encounter other religions, I will talk to them openly." (Student No.3, Female, Malay)
However there were some alternative perspectives outlined with regard to the salience of traditional practices as in the following.

"My observation of other cultural and religious practices doesn't affect the way that I perceive death to be. Because to me a person's death is more a celebration of the person's life." (Student No.7, Female, Chinese)

Similarly, a minority perspective on the relevance of religious and cultural practices was provided by two students. In the first quote below one participant emphasised the importance of individual freedom in discovering the best way to cope with bereavement while the second articulated how, for her, religious practices and beliefs held less significance.

"So what I've learned from the session is that it is up to individ-
ual to actually cope with death and different people cope differ-
ently. It is really important to find a way in which you feel
comfortable with and just go ahead with it. If you force yourself
to feel happy and try to forget about your loved one it will be
very difficult for you to move on with your life because it will
constantly haunt you." (Student No.10, Female, Malay)

"I think coping with death and bereavement really depends on individual themselves as well as culture and religious influences. However I'm different from most of you all because you all have religious practices and beliefs that are relatively stronger than mine." (Student No.13, Female, Chinese)

\section{Discomfort}

Direct observation of the group process revealed that students were respectful in the way they listened to each other's stories. They were attentive and empathic in response to overt expressions of distress. Some acknowledged difficulty in sharing the unique rituals and customs associated with their own religious and ethnic backgrounds with others from a different faith. However this seemed to be counterbalanced by the positive aspects of being able to express their feelings in a supportive atmosphere.

"I think it is actually difficult to talk about these issues in front of others sincerely. But it seems like we all have the similarities of expressing our sadness and grief. For me there is nothing much better to have the opportunity in this situation so that we know others feel the same." (Student No.2, Female, Malay)

\section{Discussion}

Most students who participated in this study identified strongly with beliefs and rituals surrounding death and bereavement anchored within their own ethnic and religious backgrounds. Those students who acknowledged religious beliefs as important were not dismissive of the contributions of those for whom religious observance held less salience. The manner in which the students listened to each other showed interest and involvement. They nodded and offered tissues to the student who though moved to 
tears was willing to share her story. The length of narratives shared by the students varied and some were more emotional in content than others. Students were not reticent about letting the emotion come through and continued their narratives to the point they wanted to, before letting the next student take over. Although the students had the opportunity to clarify what was shared, there were no clashes. Their interaction revolved around acknowledging similarities and differences in beliefs and practices without judging or challenging the narratives. Whether the students would have shared as freely, if the ground rules had not been so specific is uncertain and warrants further study. Except in a few instances, students did not make connections between their personal experience of death and bereavement and their clinical role. This might be due to the fact that the list of questions provided at the outset did not refer to implications for clinical practice. This was in part due to the authors' decision to keep the focus on the sharing of personal narratives.

The most obvious impact of the group experience on the students appeared to be their recognition that while the concrete expressions may be widely divergent, similar underlying purposes were served by the various rituals and practices described in their narratives. This is not to minimise their difficulty in openly discussing the specific details with people of a different religion, possibly an unprecedented activity for most if not all participants. However, there is a strong sense that the experience was at the least awareness raising. Also, participation in the group did not appear to precipitate persisting emotional distress insofar as the authors were not approached afterwards despite their availability.

The students acknowledged discomfort, therefore, did not seem to prevent them appreciating that they held certain transcendent beliefs and values in common with peers of a different religion and culture. Overarching themes were obvious - of death as a transition to a better life and of bereavement rituals as providing both personal comfort as well as meaningful support for the extended family. The emergence of this finding appears to resonate with $\mathrm{Ho}^{\prime}{ }^{14}$ contention that it is not difficult to extract commonalities from the major religions in the core beliefs and practices surrounding death and bereavement such that a trans-cultural applicability can be achieved.

It is also notable that the minority position of students, for whom religious rituals surrounding death held less salience, did not emerge in opposition to the majority view. Both perspectives, the one espousing cultural and religious values, the other asserting individual choice in the response to death and grief, appeared to be tolerated and did not seem to undermine the overall sense of the group as a positive and valued experience.

This may be especially relevant as one of the recognised pitfalls in implementing cultural competence curricula is that stereotypical attitudes and expectations of people from a particular cultural or ethnic background may be unwittingly reinforced. One of the important elements in culturally competent practice is that the doctor avoids global assumptions and actively clarifies the individual perspective of the patient or family involved. This may diverge considerably from the expected position as assumed from their stated religious affiliation or ethnic origin.

A further theme that emerged was how some students, even at such an early stage in their medical careers, appeared to process the experience of death and grief through the lens of their developing medical identities. There was an obvious duality of perspective on death and the circumstances surrounding it, one highly personal, emotional and subjective and the other more clinical and detached. The latter was reflected in students' use of medical terminology and in their frequently critical analysis of the treatment their loved ones has received.

The emergence of this theme would seem to suggest a particular opportunity for developing reflective learning in the subject area. One of the tasks that the doctor faces in communicating with patients and families in end of life care is to maintain an objective and rational position on one hand, while also demonstrating empathy with the patient's or family's unique emotional experience on the other. The students, in the fourth year of training, seem already to be adopting this dual perspective on death and bereavement. Taken together, our findings support the viability of small interactive group approaches with ethnically and religiously diverse medical students in the important subject area of death and bereavement as part of the curriculum. Perhaps the most important practical implication is in challenging the notion that addressing this subject area directly in a group process is fraught and therefore better avoided.

Traditional teaching methods may not help students uncover any unresolved issues on a personal level. However, interactive group discussion provides an opportunity for students to reflect on themselves and their experiences while broadening their understanding of how others cope. Important implications arise in the application to undergraduate curriculum planning. Firstly, it has been previously found that emotional openness within a group with mixed ethnic composition in part relates to the extent to which the participants feel secure and confident in the group leadership. ${ }^{17}$ This finding, and our own experience, suggests that the planning and delivery of small group teaching in this area may require some level of experience and training in group methods. Consequently faculty with necessary skills and qualifications may need to be recruited from outside the college if not available within. Secondly, in the development of a curriculum addressing the cross cultural aspects of death and bereavement, an experiential group would need to be complemented by a more skills based approach addressing common clinical scenarios and incorporating a range of techniques including role play and reflections. Finally, it would be essential that a robust 
system of evaluation be built into the planning and delivery of this curriculum from the outset so that the subsequent impact on doctor's behaviour and communication is determined.

\section{Limitations}

The study is based on a single group study with a small selfselected sample of participants. Also, two of the three authors are academic staff of the college department. Therefore it is possible that the students' responses might have been different if the study had been conducted entirely by external researchers. PMC, as a setting, may also have characteristics which limit generalisation of findings to other medical schools. In particular, our students may have been exposed to certain cultural influences that differed from their counter-parts in other Malaysian universities. They had all lived and studied in Dublin, Ireland for the initial two and a half years of their course.

A further consideration is that the group itself was relatively structured and the process managed by experienced clinician-teachers in the area of mental health. This may limit the applicability to the "real world" educational context.

\section{Conclusion}

Our findings suggest that undergraduate medical curricula in Asian multi-ethnic settings could be strengthened by facilitated groups exploring the cultural issues surrounding death and bereavement. However, careful planning is required, attending to issues of structure and process. Facilitators should possess sufficient skills and experience in group work and where necessary the involvement of external facilitators may be helpful.

\section{Conflict of Interest}

The authors declare that they have no conflict of interest.

\section{References}

1. Like R. Educating clinicians about cultural competence and disparities in health and health care. J Contin Educ Health. 2011;31(3):196-206.

2. Dogra N, Reitmanova S, Carter- Pokras O. Teaching cultural diversity: current status in UK, US and Canadian medical schools. J Gen Intern Med. 2010;25 Suppl 2:S164-8

3. Rapp D. Integrating cultural competency into the undergraduate medical curriculum. Med Educ. 2006;40:704-10.

4. Betancourt JR,Green AR. Commentary: linking cultural competence training to improved health outcomes: perspectives from the field. Acad Med. 2010;85(4):583-5.

5. Hardy-Bougere M. Cultural manifestations of grief and bereavement: a clinical perspective. J Cult Divers. 2008;15(2):66-9.

6. Holloway M. Death the great leveller? Toward a transcultural spirituality of dying and bereavement. J Clin Nurs. 2006;15:833-9.

7. Tervalon M, Murray- García J. Cultural humility versus cultural competence: a critical distinction in defining physician training outcomes in multicultural education. J Health Care Poor Underserved.1998;9(2):117-25.

8. Kumas-Tan Z, Beagan B, Loppie C, MacLeod A, Frank B. Measures of cultural competence: examining hidden assumptions. Acad Med. 2007;82(6):548-57.

9. Lopez L, Vranceanu AM, Cohen A, Betancourt J, Weissman J. Personal characteristics associated with resident physicians' self-perceptions of preparedness to deliver cross-cultural care. J Gen Intern Med. 2008;23(12): 1953-8.

10. Nordstrom A, Fjellman-Wiklund A, Grysell T. The effect of a roleplaying exercise on clerkship students' views of death notification: the Swedish experience. Int J Med Educ. 2011;2:24-9.

11. Dickinson G. Teaching end-of-life issues in US medical schools: 1975 to 2005. Am J Hosp Palliat Care. 2006;23(3):197-204

12. Wear D. "Face-to-face with it": medical students' narratives about their end of life education. Acad Med. 2002;77(4):271-7.

13. Shapiro E. Grief in family and cultural context: learning from Latino families. Cult Divers Ment Health. 1995;1(2):159-76.

14. Ho D, Ho R. Measuring spirituality and spiritual emptiness: toward ecumenicity and transcultural applicability. Review of General Psychology. 2007;11(1):62-74.

15. Graneheim UH, Lundman B. Qualitative content analysis in nursing research: concepts, procedures and measures to achieve trustworthiness. Nurse Educ Today. 2004,24:105-12.

16. Miles MB, Huberman A. Qualitative data analysis: an expanded source book. 2nd ed. London: Sage;1994.

17. Bhugra D, Gupta S, Bhui K, Craig T. WPA guidance on mental health and mental health care in migrants. World Psychiatry. 2011;10(1):2-10. 